\title{
Emergency Medical Services vs. Police: Impact of Pre-Hospital Transportation Method on Outcomes of Trauma Patients with Firearm Injuries
}

\section{Christina Wohler}

Thomas Jefferson University https://orcid.org/0000-0003-1115-3849

\section{Rachel Denneny}

Thomas Jefferson University

\section{Allegra Bermudez}

Thomas Jefferson University

\section{Robert Wilson}

Thomas Jefferson University

\section{Douglas Gouchoe}

Thomas Jefferson University

\section{Ayako Shimada}

Thomas Jefferson University

Stanton Miller ( $\nabla$ stanton.miller@jefferson.edu )

Acute Care Surgery Division, Jefferson Center for Injury Prevention \& Research https://orcid.org/00000001-5028-6949

\section{Original Contribution}

Keywords: firearms, violence, trauma

Posted Date: November 17th, 2020

DOI: https://doi.org/10.21203/rs.3.rs-103342/v1

License: (c) (1) This work is licensed under a Creative Commons Attribution 4.0 International License. Read Full License 


\section{Abstract \\ Background}

Firearms are a significant cause of morbidity and mortality in the United States. Few studies exist to investigate the impact of pre-hospital transportation methods on trauma patient outcomes.

\section{Methods}

Patients with firearm injuries were identified using an institutional trauma registry (2008 to 2017). Data on patient demographics, hospital transportation, treatments, and outcomes was collected and analyzed. Patient characteristics between Emergency Medical Services (EMS) vs. police transport groups were compared using Kruskal-Wallis, chi-square, or Fisher's exact tests as appropriate.

\section{Results}

Of 224 patients identified, 147 (66\%) were transported by EMS and 77 (34\%) were transported by police. There was no significant difference in patient demographics between groups. Most patients were male (94.2\%) and African American (69.2\%), with a mean age of 27.1 years. $84.4 \%$ of patients suffered from an externally-inflicted gunshot wound, while $9.4 \%$ of patients had inflicted the wound themselves. Handguns were the weapon most commonly used. There was no significant difference in in-hospital treatments or mortality between patients transported by EMS vs. police. $44.1 \%$ of patients underwent surgery, and $34.8 \%$ required specialist consultation. The mean hospital length of stay for all patients was 1 day, and $27.7 \%$ of all patients expired during admission.

\section{Conclusions}

There is no difference in hospital treatment or mortality between firearm victims transported by EMS vs. police.

\section{Background}

Trauma is the leading cause of death for Americans aged 1 to 44 years, ${ }^{1}$ representing an important preventable cause of mortality across the nation. Firearms are major contributors, with over 35,000 Americans dying of firearm-related injuries in $2015 .{ }^{2}$ Worldwide, $83.7 \%$ of all firearm deaths occur in the US. ${ }^{2}$ The rate of firearm homicides in the US is 24.9 times greater than that of other high-income countries, and the rate of firearm suicides is 9.8 times greater. $^{2}$

Central nervous system damage is the leading cause of trauma-related mortality, followed by intrathoracic hemorrhage. ${ }^{3}$ The highest risk of mortality occurs immediately after injury, with $61 \%$ of 
trauma victims expiring before reaching a hospital. ${ }^{4}$ Among those who are hospitalized, $7.3 \%$ of trauma patients expire before discharge, with $70 \%$ dying in the first 24 hours. ${ }^{3}$ Thus for trauma survivors, the immediate post-injury interval represents a critical target for improvements in treatment and survival.

Optimization of transportation for trauma victims is essential. Previous studies have demonstrated that despite longer transport times, trauma patients transported by helicopter have lower mortality rates than those transported by ground. 5,6 Among patients transported by ground, Emergency Medical Services (EMS) response time does not appear to significantly impact overall survival. ${ }^{7}$ Additional studies examining EMS vs. non-EMS transport indicate that non-EMS transportation is faster, but did not demonstrate any difference in mortality or in-hospital complications between groups. ${ }^{8}$

No recent studies have been conducted in the United States to compare in-hospital mortality and complications for trauma patients transported by EMS vs. non-EMS entities. This study is intended to fill that gap, and to further explore the demographics and outcomes of trauma patients in an urban Level I trauma center.

\section{Methods}

This study intends to retrospectively examine the demographic characteristics, in-hospital treatments, and outcomes of firearm victims transported by EMS vs. police. Approval for this study was granted by the appropriate health system Institutional Review Board (IRB). Using a health system trauma registry, the medical records of patients treated for firearm injuries at one urban Level I trauma center over a 10-year period (2008 to 2017) were identified. Of these patients, 224 were identified as having been transported by police or EMS, and patients transported by other means were excluded. Patient characteristics such as demographics, mechanism of injury, treatments received, and outcomes between the police and EMS transport groups were compared using Kruskal-Wallis tests, chi-square tests, or Fisher's exact tests, as appropriate. We identified 5 categories of firearm used to inflict patient injuries: pellet/BB gun, handgun, rifle, shotgun, and unknown. The significance level of all tests was set a priori to 0.05 . All analyses were performed in SAS 9.4 (SAS Institute Inc., Cary, NC).

\section{Results}

$66 \%$ of patients studied were transported by EMS, and $34 \%$ by police. Table 1 summarizes the data. The majority of patients in both the EMS and police transport groups were male (94.2\%) and black $(69.2 \%)$ with a mean age of 27 years. The majority of patients in both groups did not have health insurance $(63.8 \%)$, and of those insured, $81.3 \%$ had government insurance. There was no significant difference in the distribution of demographic characteristics between EMS and police transport groups. 
Table 1

Patient Demographics, Mode of Arrival, Mechanism of Injury, Interventions, and Mortality

\begin{tabular}{|c|c|c|c|c|}
\hline & & All $(n=224)$ & $\begin{array}{l}\text { EMS }(n=147 \\
66 \%)\end{array}$ & $\begin{array}{l}\text { Police }(n= \\
77,34 \%\end{array}$ \\
\hline Age, mean (SD) & & $27.1(11.2)$ & $27.6(12.5)$ & $26.1(8.2)$ \\
\hline \multirow[t]{2}{*}{ Sex, n (\%) } & Male & $210(94.2)$ & 137 (93.2) & $73(96.0)$ \\
\hline & Female & $13(5.2)$ & $10(6.8)$ & $3(4.0)$ \\
\hline \multirow[t]{4}{*}{ Race, n (\%) } & Black & 148 (69.2) & $94(66.7)$ & $54(74.0)$ \\
\hline & White/Hispanic & $39(18.2)$ & $31(22.0)$ & $8(11.0)$ \\
\hline & $\begin{array}{l}\text { Asian/Pacific } \\
\text { Islander }\end{array}$ & $11(5.1)$ & $7(5.0)$ & $4(5.5)$ \\
\hline & Other & $16(7.5)$ & $9(6.3)$ & $7(9.5)$ \\
\hline History of Firearm Injuries & & $23(10.3)$ & $18(12.2)$ & $5(6.5)$ \\
\hline $\begin{array}{l}\text { History of Other Violent } \\
\text { Injuries }\end{array}$ & & $32(14.3)$ & $17(11.6)$ & $15(19.5)$ \\
\hline History of Mental IIIness & & $15(6.7)$ & $9(6.1)$ & $6(7.8)$ \\
\hline \multirow[t]{4}{*}{ Mechanism of Injury } & Accidental & $20(8.9)$ & $15(10.2)$ & $5(6.5)$ \\
\hline & Intentional & 187 (83.5) & $120(81.6)$ & $67(87.0)$ \\
\hline & $\begin{array}{l}\text { Externally- } \\
\text { Inflicted }\end{array}$ & $189(84.4)$ & $116(78.9)$ & $73(94.8)$ \\
\hline & Self-Inflicted & $21(9.4)$ & $21(14.3)$ & $0(0.0)$ \\
\hline \multirow[t]{5}{*}{ Type of Gun Used } & Pellet/BB Gun & $4(1.8)$ & $3(2.0)$ & $1(1.3)$ \\
\hline & Handgun & $39(17.4)$ & $29(19.7)$ & $10(13.0)$ \\
\hline & Rifle & $1(0.4)$ & $0(0.0)$ & $1(1.3)$ \\
\hline & Shotgun & $6(2.7)$ & $5(3.4)$ & $1(1.3)$ \\
\hline & Unknown & $174(77.7)$ & $110(74.8)$ & $64(83.1)$ \\
\hline $\begin{array}{l}\text { Substance Use at Time of } \\
\text { Injury }\end{array}$ & Alcohol & $23(10.3)$ & $15(10.2)$ & 8 (10.4) \\
\hline
\end{tabular}




\begin{tabular}{|c|c|c|c|c|}
\hline & & All $(n=224)$ & $\begin{array}{l}\text { EMS }(n=147 \\
66 \%)\end{array}$ & $\begin{array}{l}\text { Police }(n= \\
77,34 \%\end{array}$ \\
\hline & \multirow[t]{14}{*}{ Illicit Drugs } & Benzodiazepines & $8(33.3)$ & $2(13.3)$ \\
\hline & & $10(25.6)$ & $7(29.2)$ & $6(40.0)$ \\
\hline & & Opiates & $1(4.2)$ & $0(0.0)$ \\
\hline & & $13(33.3)$ & $3(12.5)$ & 2 (13.3) \\
\hline & & Barbiturates & $2(8.3)$ & $2(13.3)$ \\
\hline & & $1(2.6)$ & $16(66.7)$ & $12(80.0)$ \\
\hline & & Cocaine & $1(4.2)$ & $0(0.0)$ \\
\hline & & $5(12.8)$ & & \\
\hline & & Amphetamines & & \\
\hline & & $4(10.3)$ & & \\
\hline & & Cannabis & & \\
\hline & & $28(71.8)$ & & \\
\hline & & РCP & & \\
\hline & & $1(2.6)$ & & \\
\hline \multirow[t]{5}{*}{ Insurance } & Yes & Commercial & $9(23.1)$ & $3(12.0)$ \\
\hline & & $12(18.8)$ & $30(76.9)$ & $22(88.0)$ \\
\hline & & Government & & \\
\hline & & $52(81.3)$ & & \\
\hline & No & $143(63.8)$ & $97(66.0)$ & $46(59.7)$ \\
\hline Surgical Intervention & & $97(44.1)$ & $58(40.6)$ & $39(50.7)$ \\
\hline Specialist Consultation & & $77(34.8)$ & $46(31.9)$ & $31(40.3)$ \\
\hline $\begin{array}{l}\text { Median Length of Stay } \\
\text { (days) }\end{array}$ & & 1 & 1 & 1 \\
\hline \multirow{3}{*}{$\begin{array}{l}\text { Glasgow Coma Scale } \\
\text { (GCS) }\end{array}$} & Mild (13-15) & $136(67.0)$ & $89(66.4)$ & $47(68.1)$ \\
\hline & Moderate $(9-12)$ & $5(2.5)$ & $4(3.0)$ & $1(1.5)$ \\
\hline & Severe (8 or less) & $62(30.5)$ & $41(20.6)$ & $21(30.4)$ \\
\hline Death During Admission & & $62(27.7)$ & $38(25.9)$ & $24(31.2)$ \\
\hline
\end{tabular}


In both the EMS and police transport groups, over $80 \%$ of patients' injuries were caused by intentional violence, with handguns being the weapon most commonly used (17.4\%). In the police transport group, significantly more patients' injuries were inflicted by someone other than the patient $(p<.001)$ and were more likely to be intentional $(p=0.02)$ versus accidental. More than $80 \%$ of injuries in both groups were non-accidental.

It was observed that more patients in the EMS transport group had sustained prior firearm injuries than in the police transport group ( $12.2 \%$ and $6.5 \%$, respectively), but this association was not statistically significant. $14.3 \%$ of all patients had previous violent injuries documented in their medical records. Nearly $7 \%$ of patients had a documented history of mental illness, with depression being the most commonly reported $(66.7 \%)$. Nearly $22 \%$ of all patients had a history of tobacco use. $10.3 \%$ of patients had alcohol in their system at the time of injury. $17.4 \%$ of patients' urine drug screens were positive for illicit substances, with cannabis being most common (71.8\%), followed by opiates $(33.3 \%)$ and benzodiazepines (25.6\%).

In the police transport group, around $30 \%$ of patients presented to the hospital with Glasgow Coma Scale (GCS) scores less than or equal to 8 (i.e., severe), whereas around $20 \%$ of EMS transport patients fell in this range. There was some evidence that patients transported by police had slightly higher rates of requiring specialist consultation (40.3\%) and surgical intervention (50.7\%), but the differences did not reach the level of statistical significance. Over $40 \%$ of all patients eventually required surgery, and over $30 \%$ required specialist care, with nearly $20 \%$ requiring more than one specialist. Over $70 \%$ of all patients were discharged in good health, while around $30 \%$ expired during their admission. Most patients were discharged home (88.1\%). The median length of stay was 1 day, with hospitalizations ending with either discharge or death. There was no statistically significant difference in the distributions of outcomes between EMS and police transportation groups.

\section{Discussion}

Firearm violence remains a critical public health issue in the United States, and is a target of ongoing research. Currently, there is limited data on the impact of pre-hospital transportation methods on patient outcomes in the United States. Our data suggest there is no significant difference in mortality between patients transported by EMS and police. The severity of patient injuries, indicated by GCS score on presentation, were also similar between groups. Additionally, there were no significant differences in treatment requirements or hospital length of stay between groups. Therefore, our data suggest that police transportation of trauma patients does not increase the risk of mortality or complications as compared to EMS transport, and can be considered an acceptable alternative. Thus, in situations where urgent medical treatment is required, patients may benefit from immediate police transport rather than awaiting EMS response. Further research will be needed to investigate transit times of each method, distance traveled, and other contributing factors. 
Services provided by EMS typically include initial stabilization of patients on scene, as well as support during transportation to a higher level of care. This is particularly essential in patients with a GCS less than $8,{ }^{9}$ who require intubation or other ventilatory support for airway protection. According to our data, around $30 \%$ of patients in the police transport group had a GCS less than 8 , while around $20 \%$ from the EMS transport group fell into this category. It can be assumed that the patients transported by police were not intubated prior to arrival at the hospital. Despite this lack of intervention, these patients had similar outcomes to those transported by EMS, who likely benefited from additional resuscitative measures.

There are several limitations to this study. First, patient data was extracted from an electronic medical record system, which provided limited insight into the patients' pre-hospital course. There was no data available on EMS interventions, transport time, transport distance, patient injury severity score (ISS), or explanation to support the use of one mode of transport over another. Firearm type was also infrequently reported. Further research into specific EMS interventions and the impact of transportation time on patient outcomes is needed. Finally, this study was conducted in the setting of an urban Level I trauma center, and its results may not apply in other settings.

\section{Abbreviations}

1. EMS $=$ Emergency Medical Services

2. IRB = Institutional Review Board

3. GCS = Glasgow Coma Scale

\section{Declarations}

1. Ethics Approval and Consent to Participate: This study was approved by the Thomas Jefferson University Institutional Review Board (IRB)

2. Consent for Publication: Not applicable

3. Availability of Data and Materials: The datasets used and/or analyzed during the current study are available from the corresponding author on reasonable request.

4. Competing Interests: The authors declare that they have no competing interests.

5. Funding: Not applicable

6. Author's Contributions: $\mathrm{CW}$ analyzed patient data and wrote the manuscript. RD assisted with data collection. AB assisted with data collection and manuscript editing. RW participated in data collection and statistical analysis. DG assisted with data collection. AS assisted with statistical analysis of the data. SB helped in writing and editing the manuscript.

7. Acknowledgements: Not applicable

\section{References}


1. Web-Based Injury Statistics Query and Reporting System. Centers for Disease Control and Prevention. Atlanta, GA. http://www.cdc.gov/injury/wisqars. Accessed March 30, 2020.

2. Grinshteyn E, Hemenway D. Violent Death Rates in the US Compared to Those of Other High-Income Countries, 2015. Preventive Medicine. 2019; 23:20-26.

3. Acosta JA, Yang JC, Winchell RJ, Simons RK, Fortlage DA, Hollingsworth-Fridlund P, Hoyt DB. Lethal Injuries and Time to Death in a Level I Trauma Center. Journal of the American College of Surgeons. 1998; 186(5):528-533.

4. Gunst M, Ghaemmaghami V, Gruszecki A, Urban J, Frankel H, Shafi S. Changing Epidemiology of Trauma Deaths Leads to a Bimodal Distribution. Baylor University Medical Center Proceedings. 2010; 23(4):349-354.

5. Buchanan IM, Coates A, Sne N. Does Mode of Transport Confer a Mortality Benefit in Trauma Patients? Characteristics and Outcomes at an Ontario Lead Trauma Hospital. Canadian Journal of Emergency Medicine. 2016; 18(5):363-369.

6. Mitchell AD, Tallon JM, Sealy B. Air Versus Ground Transport of Major Trauma Patients to a Tertiary Trauma Centre: A Province-Wide Comparison Using TRISS Analysis. Canadian Journal of Surgery. 2007; 50(2):129-133.

7. Blanchard IE, Doig CJ, Hagel BE, Anton AR, Zygun DA, Kortbeek JB, Powell DG, Williamson TS, Fick GH, Innes GD. Emergency Medical Services Response Time and Mortality in an Urban Setting. Prehospital Emergency Care. 2012; 16(1):142-151.

8. Cornwell EE III, Belzberg H, Hennigan K, Maxson C, Montoya G, Rosenbluth A, Velmahos GC, Berne TC, Demetriades D. Emergency Medical Services (EMS) vs. Non-EMS Transport of Critically Injured Patients: A Prospective Evaluation. JAMA Surgery. 2000; 135(3):315-319.

9. Gnugnoli DM, Richards E, Shafer K. EMS, Field Intubation. In: StatPearls. 2020. https://www.ncbi.nlm.nih.gov/books/NBK538221/. Accessed August 25, 2020. 